EPJ manuscript No.

(will be inserted by the editor)

\title{
Nonequilibrium properties of an atomic quantum dot coupled to a Bose-Einstein condensate
}

\author{
${\text { Roberta } \text { Citro }^{1, a} \text {, Adele Naddeo }}^{2, b}$, and Francesco Romeo ${ }^{1, c}$ \\ 1 Dipartimento di Fisica "E. R. Caianiello", Universitá degli Studi di Salerno and CNR- \\ SPIN, Unitá Operativa di Salerno, Via Ponte Don Melillo, 84084 Fisciano (SA), Italy \\ 2 Dipartimento di Fisica "E. R. Caianiello", Universitá degli Studi di Salerno, and CNISM, \\ Unitá di Ricerca di Salerno, Via Ponte Don Melillo, 84084 Fisciano (SA), Italy
}

\begin{abstract}
We study nonequilibrium properties of an atomic quantum $\operatorname{dot}(\mathrm{AQD})$ coupled to a Bose-Einstein condensate (BEC) within KeldyshGreen's function formalism when the AQD level is varied harmonically in time. Nonequilibrium features in the AQD energy absorption spectrum are the side peaks that develop as an effect of photon absorption and emission. We show that atoms can be efficiently transferred from the BEC into the AQD for the parameter regime of current experiments with cold atoms.
\end{abstract}

\section{Introduction}

The tremendous experimental progress in the field of ultracold atoms has made possible with unprecedent control the realization and design of many-body atomic physics by means of very stable optical lattices and by the use of Feshbach resonances [1] 2. That has triggered the study of such systems for quantum information processing purposes 3 and as quantum simulators of condensed matter Hamiltonians 4. In this context the experimental development of atom chips 5] opened new perspectives towards atomic mesoscopic physics. Indeed the possibility to build up atomic waveguides above microfabricated surfaces allows one to study a variety of phenomena such as the interplay between interaction and transport through point contacts 6 , the dynamics of soliton-like structures in waveguides [7] and the coherent flow of a Bose Einstein condensate (BEC) through a double barrier potential realized as a quantum dot in a magnetic waveguide 8 .

Another route towards trapping and manipulation of single atoms 9 has been recently proposed, which relies on a focused laser beam superimposed to a trap holding an atomic Bose Einstein condensate; that leads to the formation of an atomic quantum dot (AQD) 10, i.e. a single atom in a tight trap, which is coupled to a superfluid reservoir via laser transitions. In particular, in the case of a one dimensional superfluid reservoir, the system maps onto a spin-boson model with ohmic coupling, which

\footnotetext{
${ }^{a}$ e-mail: citro@sa.infn.it

b e-mail: naddeo@sa.infn.it

c e-mail: fromeo@sa.infn.it
} 
exhibits a dissipative quantum phase transition 11 and provides a spectroscopic tool to engineer open quantum systems and study their irreversible dynamics [12. Such a system can also be employed as a probe of BEC phase fluctuations via dephasing measurements of the internal states of the AQD, as discussed in [13. More recently an AQD coherently coupled by optical transitions to two BEC reservoirs has also been considered together with a Josephson tunneling between the two BECs 14. Finally, a bosonic single-impurity Anderson model (AM) has been recently numerically investigated [15] in order to understand the local dynamics of an AQD coupled to a BEC system, accounting for the entanglement and the decoherence of the macroscopic condensate. In general, the strong collisional interaction in the AQD can locally break a BEC state to bring up the excitations of normal particles inside the dot and thus a non-equilibrium particle transport can be realized, while the number of excited particles in the QD gives an indirect probe of the coherence of the macroscopic condensate.

In this work we discuss the realization of coherent particle transport in an AQD resonantly coupled to a BEC via a Raman transition with effective Rabi frequency and realized by harmonic time modulation of the AQD level. We do consider a fully quantum non-equilibrium situation within a Keldysh approach, not simply looking at the stationary limit, and focus on the local spectral properties of the QD.

\section{Model and results}

In our model system we consider cold bosonic atoms with two hyperfine ground states $a$ and $b$. Atoms in state $a$, which form the reservoir, are confined in a shallow trapping potential $V_{a}(\mathbf{x})$ while atoms in state $b$ are localized in a tightly confining potential $V_{b}(\mathbf{x})$. The atoms are identical bosons of mass $m$ and form a Bose-Einstein condensate in a state $a$, i.e. a quantum reservoir. Furthermore states $a$ and $b$ are coupled via a Raman transition with effective Rabi frequency $\Omega$. In the collisional blockade limit for atoms $b$ only a single atom is localized on the dot. Atoms $a$ and $b$ are described by the field operators $\widehat{\Psi}_{a}$ and $\widehat{\Psi}_{b}$, with $\widehat{\Psi}_{b}(\mathbf{x})=\psi_{b}(\mathbf{x}) \widehat{b}$, where the operator $\widehat{b}$ destroys an atom $b$ in the dot in the lowest vibrational state with wave function $\psi_{b}(\mathbf{x})$ and $\widehat{\Psi}_{a}(\mathbf{x}) \sim \widehat{\rho}_{a}(\mathbf{x})^{1 / 2} e^{-i \widehat{\phi}(\mathbf{x})}$ in the long-wavelength limit, where $\hat{\rho}_{a}$ and $\hat{\phi}$ are the density and phase operator, respectively. The general many-body Hamiltonian of our system is 10:

$$
H=H_{a}+H_{b}+H_{a b}
$$

where

$$
H_{a}=\frac{1}{2} \int d \mathbf{x}\left(\frac{\hbar^{2}}{m} \rho_{s}|\nabla \widehat{\phi}|^{2}(\mathbf{x})+\frac{m v_{s}^{2}}{\rho_{a}} \widehat{\Pi}^{2}(\mathbf{x})\right)
$$

and

$$
\begin{aligned}
& H_{b}+H_{a b}=\left(-\hbar \delta_{0}+g_{a b} \int d \mathbf{x}\left|\psi_{b}(\mathbf{x})\right|^{2} \widehat{\rho}_{a}(\mathbf{x})\right) \widehat{b}^{+} \widehat{b}+ \\
& \frac{U_{b b}}{2} \widehat{b}^{+} \widehat{b}^{+} \widehat{b b}+\hbar \Omega(t) \int d \mathbf{x} \cos (\mathbf{k} \cdot \mathbf{x})\left(\widehat{\Psi}_{a}(\mathbf{x}) \widehat{\Psi}_{b}^{\dagger}(\mathbf{x}) e^{i \omega_{p} t}+\text { h.c. }\right),
\end{aligned}
$$

where the first term in Eq. (3) contains the Raman detuning $\delta_{0}$ and the collisional interactions between the dot and the reservoir, $g_{a b}=\frac{4 \pi \hbar^{2} a_{a b}}{m}$, with effective mass $m=m_{a} m_{b} /\left(m_{a}+m_{b}\right)$ and $s$-wave scattering lengths $a_{a b} ; \widehat{\rho}_{a}(\mathbf{x})=\widehat{\Psi}_{a}^{+}(\mathbf{x}) \widehat{\Psi}_{a}(\mathbf{x})$ is the density operator for atoms $a$, which can be expressed in terms of the density fluctuation operator $\widehat{\Pi}$ (canonically conjugated to $\widehat{\phi}$ ) as $\widehat{\rho}_{a}(\mathbf{x})=\rho_{a}+\widehat{\Pi}(\mathbf{x})$; the 
second term describes the interaction strength of two-body s-wave collision $U_{b b} \propto g_{b b}$ between the dot atoms (which we do consider in the collisional regime) while the third one is the laser induced coupling between atoms $a$ and $b$ with Rabi frequency $\Omega$. We do consider a step-like pulse with frequency $\omega_{p}$ and duration $\tau$. Since in the collisional blockade limit $\omega_{\text {gap }} \tau \gg 1$ where $\omega_{\text {gap }}$ is the gap between the single and two-atom, the dynamics describing the coupling to all other bound states can be neglected and the laser resonantly couples the condensate with the single atom ground states of the dot with an effective strength $V_{\mathbf{k}}=\hbar \Omega \int d \mathbf{x} \cos (\mathbf{k x}) \psi_{a}(\mathbf{x}) \psi_{b}(\mathbf{x})$, i.e. the Fourier transform of the wavefunctions overlap. At low enough temperatures, the reservoir Hamiltonian (2) is that of a Bose superfluid with equilibrium density $\rho_{a}$, superfluid density $\rho_{s}$ and low energy phonon excitations of linear dispersion $\omega=v_{s}|\mathbf{q}|, v_{s}$ being the sound velocity. Eq. (2) can also be written as a bath of harmonic sound modes:

$$
H_{a}=\hbar v_{s} \sum_{\mathbf{q}}|\mathbf{q}| b_{\mathbf{q}}^{+} b_{\mathbf{q}}
$$

where $b_{\mathbf{q}}$ are standard phonon operators, defined by:

$$
\begin{gathered}
\widehat{\phi}(\mathbf{x})=i \sum_{\mathbf{q}}\left|\frac{m v_{s}}{2 \hbar \mathbf{q} V \rho_{a}}\right|^{1 / 2} e^{i \mathbf{q} \cdot \mathbf{x}}\left(b_{\mathbf{q}}-b_{-\mathbf{q}}^{+}\right) \\
\widehat{\Pi}(\mathbf{x})=\sum_{\mathbf{q}}\left|\frac{\hbar \mathbf{q} \rho_{a}}{2 m v_{s} V}\right|^{1 / 2} e^{i \mathbf{q} \cdot \mathbf{x}}\left(b_{\mathbf{q}}+b_{-\mathbf{q}}^{+}\right)
\end{gathered}
$$

$V$ being the system volume. The Hamiltonian (1) can be reduced to that of a spinboson model [11] in the collisional blockade limit (i.e. large strength $U_{b b}$ ) when the internal state of the AQD is described by a pseudospin 1/2 with the spin-up and spin-down state corresponding to single and no atom occupation respectively. Thus the following replacements can be taken: $\widehat{b}^{+} \widehat{b} \rightarrow \frac{\left(1+\hat{\sigma}_{z}\right)}{2}$ and $\widehat{b}^{+} \rightarrow \hat{\sigma}_{+}$, where $\hat{\sigma}_{+, z}$ stand for spin operators.

In order to describe the density fluctuations regime and to conserve the total number of bosons, the previous model can be conveniently mapped onto a bosonic single-impurity Anderson model (AM) [15]:

$$
H=\varepsilon b^{+} b+\frac{U}{2} b^{+} b\left(b^{+} b-1\right)+\sum_{\mathbf{k}} \varepsilon_{\mathbf{k}} b_{\mathbf{k}}^{+} b_{\mathbf{k}}+\sum_{\mathbf{k}} V_{\mathbf{k}}(t)\left(b^{+} b_{\mathbf{k}}+b_{\mathbf{k}}^{+} b\right)
$$

where $b_{\mathbf{k}}, b_{\mathbf{k}}^{+}$are annihilation and creation operators of noninteracting bosons confined in a shallow potential, $\varepsilon, U$ are immediately recognized as a function of the detuning and the collisional energies of (3),$\varepsilon_{\mathbf{k}}=v_{s}|\mathbf{k}|$ and the last term is the laser-induced hybridization between particles in the AQD and the bosonic bath where $V_{\mathbf{k}}$ has been given above. An estimate of the Hamiltonian parameters is the following [16]: the reservoir can be made of a condensate of $N \simeq 10^{3}{ }^{87} R b$ atoms with density $n_{b}=3 \cdot 10^{13}$ atoms $/ \mathrm{cm}^{3}$ in a harmonic trap (with trapping frequency $\nu_{b}=2 \pi \times 100 \mathrm{~Hz}$ ), while the tweezers trap for the AQD in the collisional regime should have frequencies of the order of hundreds of $k \mathrm{~Hz}$ and we take $\nu_{a}=2 \pi \times 100 \mathrm{kHz}$ and a detuning $\delta_{0}=284 \mathrm{kHz}$. The Raman coupling between the dot and the reservoir for these parameters can be estimated of the order of $\Omega \simeq 30 \mathrm{kHz}$.

Since we are interested in the nonequilibrium properties and particle transport through the AQD, we do consider a situation in which the AQD level is harmonically varied in time via the coupling to the radiation, i.e. $\epsilon \rightarrow \epsilon(t)=\epsilon_{0}+\epsilon_{\omega} \cos \omega t$, and employ the real-time Keldysh formalism to calculate the spectral properties of the AQD and particle transport through it. Since in the following we are interested in the 
collisional blockade limit of the AQD, the dot levels $\epsilon_{0}$ and $\epsilon_{\omega}$ have to be considered not as bare ones, but as renormalized by the requirements of a single atom occupation.

The BEC energy absorption and the particle current flowing through the AQD are given by:

$$
\begin{aligned}
\left\langle\dot{E}_{\mathbf{k}}(t)\right\rangle & =-\frac{i}{\hbar}\left\langle\left[E_{\mathbf{k}}, H(t)\right]\right\rangle, \\
J(t) & =-\left\langle\frac{d N}{d t}\right\rangle=\frac{-i}{\hbar}\langle[H, N]\rangle
\end{aligned}
$$

where $E_{\mathbf{k}}=\varepsilon_{\mathbf{k}} b_{\mathbf{k}}^{+} b_{\mathbf{k}}$ is the BEC Hamiltonian and $N=\sum_{\mathbf{k}} b_{\mathbf{k}}^{+} b_{\mathbf{k}}$. By computing the commutators, both the BEC energy absorption and the particle current can be expressed in terms of the lesser Green's function $G_{0, \mathbf{k}}^{<}\left(t, t^{\prime}\right) \equiv-i\left\langle b_{\mathbf{k}}^{+}\left(t^{\prime}\right) b(t)\right\rangle$ as:

$$
\begin{aligned}
\left\langle\dot{E}_{\mathbf{k}}(t)\right\rangle & =\varepsilon_{\mathbf{k}} J_{\mathbf{k}}, \\
J(t) & =\sum_{\mathbf{k}} J_{\mathbf{k}}
\end{aligned}
$$

where

$$
J_{\mathbf{k}}=-\frac{2}{\hbar} R e\left\{V_{\mathbf{k}}(t) G_{0, \mathbf{k}}^{<}(t, t)\right\}
$$

is the momentum resolved current. By means of the equations of motion method, a general expression for the contour ordered Green function can be found in terms of the AQD and BEC Green's function, $G$ and $g_{\mathbf{k}}$ respectively:

$$
G_{0, \mathbf{k}}\left(t, t^{\prime}\right)=\int d t_{1} G\left(t, t_{1}\right) V_{\mathbf{k}}^{*}\left(t_{1}\right) g_{\mathbf{k}}\left(t_{1}, t^{\prime}\right)
$$

By applying the Langreth rules [17] and using the explicit expression for the BEC Green's function, i.e. $g_{\mathbf{k}}^{<}\left(t, t^{\prime}\right)=i f_{B E}\left(\varepsilon_{\mathbf{k}}\right) e^{-i \varepsilon_{\mathbf{k}}\left(t-t^{\prime}\right)}$, and $g_{\mathbf{k}}^{r, a}\left(t, t^{\prime}\right)=$ $=\mp i \theta\left( \pm t \mp t^{\prime}\right) e^{-i \varepsilon_{\mathbf{k}}\left(t-t^{\prime}\right)}$, where $f_{B E}$ is the Bose distribution function of the reservoir, one can rewrite the momentum resolved particle current as:

$$
J_{\mathbf{k}}(t)=-\frac{2}{\hbar} \operatorname{Im}\left\{V_{\mathbf{k}}(t) \int_{-\infty}^{t} d t_{1} V_{\mathbf{k}}^{*}\left(t_{1}\right) e^{i \varepsilon_{\mathbf{k}}\left(t-t_{1}\right)}\left[G^{r}\left(t, t_{1}\right) f_{B E}\left(\varepsilon_{\mathbf{k}}\right)+G^{<}\left(t, t_{1}\right)\right]\right\}
$$

In terms of the self-energies the momentum resolved particle current becomes:

$$
J_{\mathbf{k}}(t)=-\frac{2}{\hbar} R e\left\{\int_{-\infty}^{t} d t_{1}\left[G^{r}\left(t, t_{1}\right) \Sigma_{\mathbf{k}}^{<}\left(t_{1}, t^{\prime}\right)+G^{<}\left(t, t_{1}\right) \Sigma_{\mathbf{k}}^{a}\left(t_{1}, t^{\prime}\right)\right]\right\}
$$

where the advanced and lesser self-energies of the reservoir are $\Sigma_{\mathbf{k}}^{a}\left(t_{1}, t^{\prime}\right)=$ $=\left|V_{\mathbf{k}}\right|^{2} g_{\mathbf{k}}^{a}\left(t_{1}, t^{\prime}\right)$ and $\Sigma_{\mathbf{k}}^{<}\left(t_{1}, t^{\prime}\right)=\left|V_{\mathbf{k}}\right|^{2} g_{\mathbf{k}}^{<}\left(t_{1}, t^{\prime}\right)$.

When the dot level is varied adiabatically in time, i.e. $\varepsilon(t)=\varepsilon_{0}+\varepsilon_{\omega} \cos (\omega t)$ with $\varepsilon_{\omega} \ll \varepsilon_{0}$, the time dependence of the tunneling $V_{\mathbf{k}}(t)$ can be neglected and $\left|V_{\mathbf{k}}\right|^{2} \approx(\hbar \Omega)^{2} e^{-\left(\sigma a \mathbf{k}^{2} / 2\right)}$, where $\sigma$ and $a$ are the ground state size of the dot and the BEC in the loosely confined direction, respectively. Let us note that, since $\sigma$ should 
be larger than the mean free distance between the particles $\sigma \gg l$ and $l \sim \xi$ where $\xi$ is the healing length $\xi=\hbar / m c$, it follows that $\sigma \gg \xi$ and we take it equal to $10^{-6} \mathrm{~m}$ while $a$ is taken $10^{-4} \mathrm{~m}[13$. In general, for very tightly confined AQD the $\mathbf{k}$ dependence of $V_{\mathbf{k}}$ can be neglected.

In this nonequilibrium problem all Green's functions and self-energies depend explicitly on two time variables, so one needs to resort to two time Fourier transform and Eq. (12) becomes:

$$
\begin{aligned}
J_{\mathbf{k}}(t)= & -\frac{2}{\hbar} \operatorname{Re}\left\{\int \frac { d E _ { 1 } d E _ { 2 } d E _ { 3 } } { ( 2 \pi ) ^ { 3 } } e ^ { i ( E _ { 3 } - E _ { 1 } ) t } \left[G^{r}\left(E_{1}, E_{2}\right) \Sigma^{<}\left(E_{2}, E_{3}\right)\right.\right. \\
& \left.\left.+G^{<}\left(E_{1}, E_{2}\right) \Sigma_{\mathbf{k}}^{a}\left(E_{2}, E_{3}\right)\right]\right\},
\end{aligned}
$$

The lesser and retarded Green function for the $\operatorname{dot} G^{<, r}\left(E_{1}, E_{2}\right)$ are given by:

$$
\begin{gathered}
G^{<}\left(E_{1}, E_{2}\right)=\sum_{\mathbf{k}} \int \frac{d \xi_{1} d \xi_{2}}{(2 \pi)^{2}} G^{r}\left(E_{1}, \xi_{1}\right) \Sigma_{\mathbf{k}}^{<}\left(\xi_{1}, \xi_{2}\right) G^{a}\left(\xi_{2}, E_{2}\right), \\
G^{r}\left(E_{1}, E_{2}\right)=\widetilde{G}^{r}\left(E_{1}, E_{2}\right)+\sum_{\mathbf{k}} \int \frac{d E_{3} d E_{4}}{(2 \pi)^{2}} \widetilde{G}^{r}\left(E_{1}, E_{3}\right) \Sigma_{\mathbf{k}}^{r}\left(E_{3}, E_{4}\right) \widetilde{G}^{r}\left(E_{4}, E_{2}\right),
\end{gathered}
$$

where the zeroth-order dot Green's function in the collisional blockade limit is $\widetilde{G}^{r}\left(t, t_{1}\right)=-i \theta\left(t-t_{1}\right) e^{-i \int_{t_{1}}^{t} d t^{\prime} \varepsilon\left(t^{\prime}\right)}=-i \theta\left(t-t_{1}\right) e^{-i \int_{t_{1}}^{t} d t^{\prime}\left[\varepsilon_{0}+\varepsilon_{\omega} \cos \left(\omega t^{\prime}\right)\right]}$ while the reservoir self-energies $\Sigma^{<}=\sum_{\mathbf{k}} \Sigma_{\mathbf{k}}^{<}$and $\Sigma^{r}=\sum_{\mathbf{k}} \Sigma_{\mathbf{k}}^{r}$ are, respectively:

$$
\begin{gathered}
\Sigma^{<}\left(E_{1}, E_{2}\right)=\sum_{\mathbf{k}}\left|V_{\mathbf{k}}\right|^{2} g_{\mathbf{k}}^{<}\left(E_{1}, E_{2}\right) \\
=-i(2 \pi)^{2} \sum_{\mathbf{k}} f_{B E}\left(\varepsilon_{\mathbf{k}}\right)\left|V_{\mathbf{k}}\right|^{2} \delta\left(E_{1}-\varepsilon_{\mathbf{k}}\right) \delta\left(\varepsilon_{\mathbf{k}}-E_{2}\right), \\
\Sigma^{r}\left(E_{1}, E_{2}\right)=\sum_{\mathbf{k}}\left|V_{\mathbf{k}}\right|^{2} g_{\mathbf{k}}^{r}\left(E_{1}, E_{2}\right) \\
=-(2 \pi) \sum_{\mathbf{k}}\left|V_{\mathbf{k}}\right|^{2} \frac{\delta\left(E_{1}-E_{2}\right)}{E_{1}-\varepsilon_{\mathbf{k}}+i 0^{+}}=\Lambda\left(E_{1}\right)-i \Gamma\left(E_{1}\right),
\end{gathered}
$$

where we have defined $\Lambda\left(E_{1}\right)$ and $\Gamma\left(E_{1}\right)$ as the principal value and imaginary part of $1 /\left(E_{1}-\epsilon_{\mathbf{k}}+i 0^{+}\right)$. By substituting the expression of $\tilde{G}^{r}$ and the self-energy in Eqs. (15) and (16) and taking the limit $\frac{\varepsilon_{\omega}}{\omega}<<1$, and keeping only terms linear in $\omega$ we get the following dot Green's functions, within single photon approximation:

$$
G^{r}\left(E_{1}, E_{2}\right)=2 \pi\left[A_{1} \delta\left(E_{1}-E_{2}\right)+B_{1}^{+} \delta\left(E_{1}-E_{2}+\omega\right)+B_{1}^{-} \delta\left(E_{1}-E_{2}-\omega\right)\right]
$$

and

$$
\begin{aligned}
G^{<}\left(E_{1}, E_{2}\right)= & i \Gamma\left(E_{2}\right)\left[f_{B E}\left(E_{1}\right) A_{1} A_{2}^{*} \delta\left(E_{2}-E_{1}\right)+A_{1}\left(B_{2}^{+}\right)^{*} \delta\left(E_{2}-E_{1}+\omega\right)+\right. \\
& A_{1}\left(B_{2}^{-}\right)^{*} \delta\left(E_{2}-E_{1}-\omega\right)+B_{1}^{+} A_{2}^{*} \delta\left(E_{2}-E_{1}-\omega\right)+ \\
& \left.B_{1}^{-} A_{2}^{*} \delta\left(E_{2}-E_{1}+\omega\right)\right]
\end{aligned}
$$

where we have defined:

$$
A_{1,2}=\frac{\left(J_{0}\left(\frac{\varepsilon \omega}{\omega}\right)\right)^{2}}{E_{1}-\varepsilon_{0}-\Lambda\left(E_{1,2}\right)+i \Gamma\left(E_{1,2}\right)}, B_{1,2}^{ \pm}=\frac{\omega J_{1}\left(\frac{\varepsilon \omega}{\omega}\right) J_{0}\left(\frac{\varepsilon \omega}{\omega}\right)}{\left(E_{1,2}-\varepsilon_{0}-\Lambda\left(E_{1,2}\right)+i \Gamma\left(E_{1,2}\right)\right)\left(E_{1,2} \pm \omega-\varepsilon_{0}-\Lambda\left(E_{1,2}\right)+i \Gamma\left(E_{1,2}\right)\right)} .
$$


Here $J_{0}\left(\frac{\varepsilon_{\omega}}{\omega}\right)$ and $J_{1}\left(\frac{\varepsilon_{\omega}}{\omega}\right)$ are the 0 -th and 1-th order Bessel functions, respectively. By inserting Eqs. (19)-(20) in Eq. (14), we can numerically evaluate both the energy absorption rate (EAR) and the particle current. The behavior of the EAR and the particle current, within single photon approximation, are shown in Figs. 1 and 2.

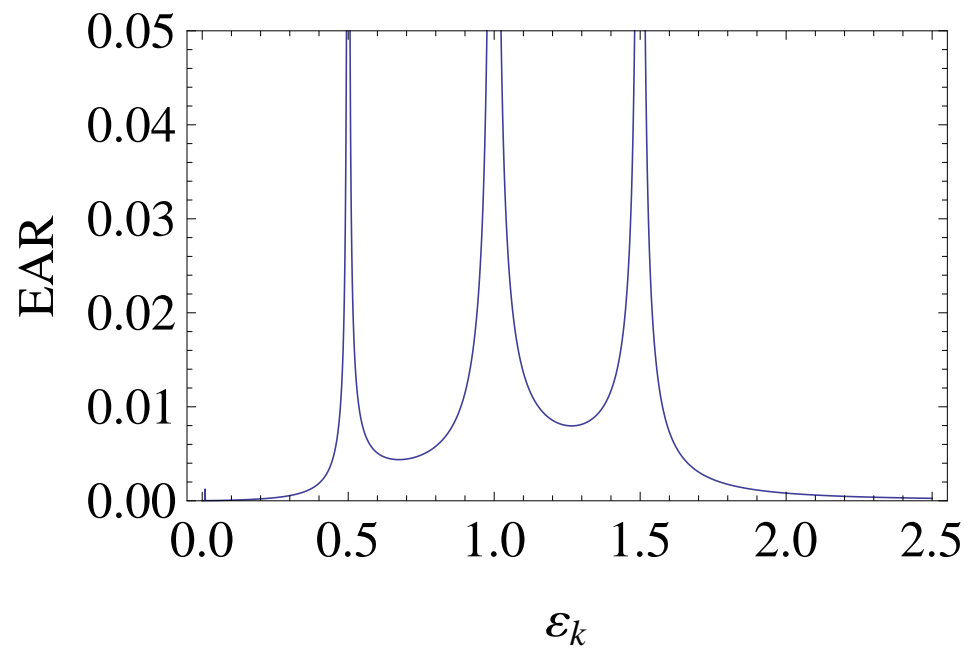

Fig. 1. Energy absorption rate as a function of $\epsilon_{k}$ for the following values of parameters (in unit of the quantum dot energy level): $\epsilon_{0}=1.0, \Omega=0.1, \epsilon_{\omega}=0.06, \omega=0.5, T=1$. The time is fixed at $t=1.0$ in units of the inverse of $\omega$.

As shown, the EAR spectrum shows a peak at $\epsilon_{0}$ and two side peaks corresponding to the absorption and emission of a photon with energy $\omega$.

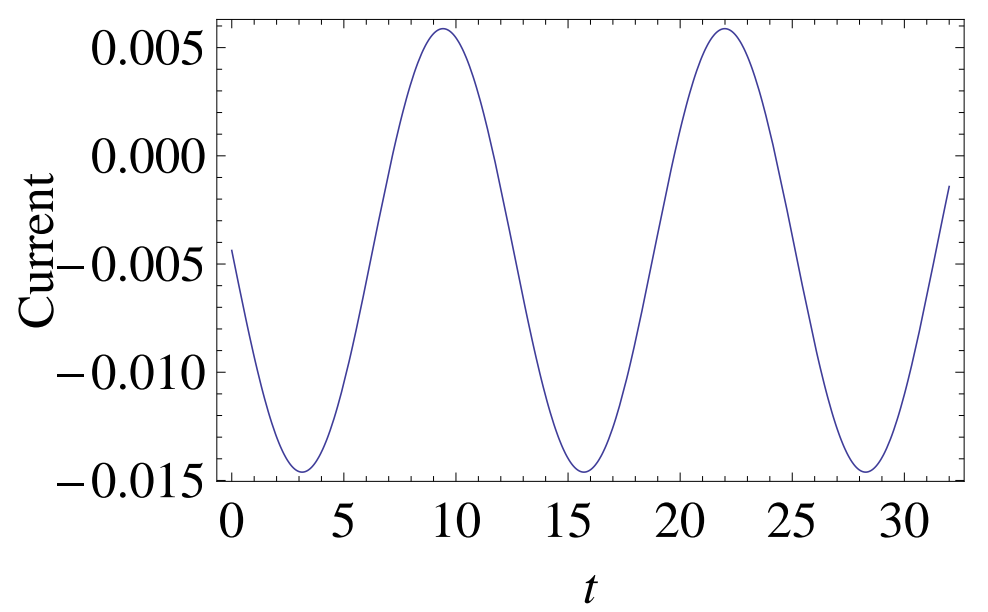

Fig. 2. Particle current as a function of time (in units of $\omega^{-1}$ ) for the following values of parameters (in unit of the quantum dot energy level): $\epsilon_{0}=1.0, \Omega=0.1, \epsilon_{\omega}=0.06, \omega=0.5$, $T=1$. 
The current induced by the quantum dot modulation follows the harmonic variation of the perturbation and its amplitude and mean value get modified as a result of single-photon absorption and emission processes.

\section{Conclusions}

The nonequilibrium properties of an atomic quantum dot (AQD) coupled to a BoseEinstein condensate (BEC) were studied within the Keldysh-Green's function formalism when the AQD level is varied harmonically in time. We have analyzed both the current and the AQD energy absorption spectrum in the full nonequilibrium situation, within a single-photon approximation and shown that it is possible to achieve an efficient way of transferring particles from the BEC to the AQD in a range of parameters interesting for current experiments with cold atoms. Both quantities are actually a subject of active investigation theoretically as well as experimentally in order to probe and manipulate such systems.

Indeed the coherent tunneling of particles from the BEC to the AQD could be employed to extract atoms on demand from the quantum reservoir, thus realizing a quantum tweezer[16]. This high degree of control is needed in several protocols for quantum information processing with neutral atoms, in particular at the initialization stage of a quantum register [18. Further applications can be envisaged, which run from the optimal control of atoms with microwave potentials for the implementation of quantum gates on an atom chip [19] to the realization of an efficient procedure to filter out from an optical lattice a preselected number of atoms per site [20. In this way lattices with a desired site occupation could be engineered.

On the other hand, the energy absorption rate could be employed as a spectroscopic tool to probe several properties of the bosonic system under study, and in particular the current autocorrelation function, in analogy with a recent proposal by Giamarchi and co-workers 21. That allows one to directly probe the frequency dependent conductivity of the system.

\section{References}

1. I. Bloch, J. Phys. B 38, (2005) S629; I. Bloch, Nature Phys. 1, (2005) 23.

2. S. Inouye, M. R. Andrews, J. Stenger, H. J. Miesner, D. M. Staper-Kurn, W. Ketterle, Nature 392, (1998) 151.

3. D. Jaksch, H. J. Briegel, J. I. Cirac, C. W. Gardiner, P. Zoller, Phys. Rev. Lett. 82, (1999) 1975.

4. D. Jaksch, P. Zoller, Ann. Phys. 315, (2005) 52; I. Bloch, J. Dalibard, W. Zwerger, Rev. Mod. Phys. 80,(2008) 885.

5. R. Folman, P. Kruger, D. Cassettari, B. Hessmo, T. Maier, J. Schmiedmayer, Phys. Rev. Lett. 84, (2000) 4749; H. Ott, J. Forthag, G. Schlotterbeck, A. Grossmann, C. Zimmermann, Phys. Rev. Lett. 87, (2001) 230401; W. Hansel, P. Hommelhoff, T. W. Hansch, J. Reichel, Nature 413, (2001) 498.

6. J. H. Thywissen, R. M. Westervelt, M. Prentiss, Phys. Rev. Lett. 83, (1999) 3762.

7. S. Komineas, N. Papanicolaou, Phys. Rev. Lett. 89, (2002) 070402.

8. T. Paul, K. Richter, P. Schlagheck, Phys. Rev. Lett. 94, (2005) 020404.

9. R. B. Diener, B. Wu, M. G. Raizen, Q. Niu, Phys. Rev. Lett. 89, (2002) 070401.

10. A. Recati, P. O. Fedichev, W. Zwerger, J. von Delft, P. Zoller, Phys. Rev. Lett. 94, (2005) 040404.

11. A. J. Leggett, S. Chakravarty, A. T. Dorsey, M. P. A. Fischer, A. Garg, W. Zwerger, Rev. Mod. Phys. 59, (1987) 1.

12. M. A. Cirone, G. De Chiara, G. M. Palma, A. Recati, New. J. Phys. 11, (2009) 103055. 
13. M. Bruderer, D. Jaksch, New. J. Phys. 8, (2006) 87.

14. I. Bausmerth, U. R. Fischer, A. Posazhennikova, Phys. Rev. A 75, (2007) 053605; U. R. Fischer, C. Iniotakis, A. Posazhennikova, Phys. Rev. A 77, (2008) 031602(R).

15. H. J. Lee, R. Bulla, Eur. Phys. J. B 56, (2007) 199; H. J. Lee, K. Byczuk, R. Bulla, Phys. Rev. B 82, (2010) 054516.

16. B. Mohring, M. Bienert, F. Haug, G. Morigi, W. P. Schleich, M. G. Raizen, Phys. Rev. A 71, (2005) 053601; S. Zippilli, B. Mohring, E. Lutz, G. Morigi, W. Schleich, Phys. Rev. A 83, (2011) 051602(R).

17. H. Haug, A. P. Jauho, Qunatum Kinetics in Transport and Optics of Semiconductors, Springer, Berlin and New York 1996.

18. T. Grunzweig, A. Hilliard, M. McGovern, M. F. Andersen, Nat. Phys. 6, 951 (2010); R. Gehr, J. Volz, G. Dubois, T. Steinmetz, Y. Colombe, B. L. Lev, R. Long, J. Esteve, J. Reichel, Phys. Rev. Lett. 104, 203602 (2010); F. Serwane, G. Zorn, T. Lompe, T. B. Ottenstein, A. N. Wenz, S. Jochim, Science 332, 336 (2011).

19. P. Treutlein, T. W. Hansch, J. Reichel, A. Negretti, M. A. Cirone, T. Calarco, Phys. Rev. A 74, (2006) 022312.

20. G. M. Nikolopoulos, D. Petrosyan, J. Phys. B: At. Mol. Opt. Phys. 43, (2010) 131001. 21. A. Iucci, M. A. Cazalilla, A. F. Ho, T. Giamarchi, Phys. Rev. A 73, (2006) 041608; C. Kollath, A. Iucci, T. Giamarchi, W. Hofstetter, U. Schollwock, Phys. Rev. Lett. 97, 050402 (2006); A. Tokuno, T. Giamarchi, Phys. Rev. Lett. 106, 205301 (2011). 